\title{
BMJ Open Think-aloud study about the diagnosis of chronic heart failure in Belgian general practice
}

\author{
Miek Smeets, ${ }^{1}$ Pieter De Witte, ${ }^{1}$ Sanne Peters, ${ }^{1}$ Bert Aertgeerts, ${ }^{1}$ \\ Stefan Janssens, ${ }^{2}$ Bert Vaes ${ }^{1,3}$
}

To cite: Smeets M, De Witte $P$, Peters S, et al. Think-aloud study about the diagnosis of chronic heart failure in Belgian general practice. BMJ Open 2019;9:e025922. doi:10.1136/ bmjopen-2018-025922

- Prepublication history and additional material for this paper are available online. To view these files, please visit the journal online (http://dx.doi. org/10.1136/bmjopen-2018025922).

MS and PDW contributed equally.

Received 8 August 2018

Revised 17 November 2018

Accepted 6 February 2019

Check for updates

(C) Author(s) (or their employer(s)) 2019. Re-use permitted under CC BY-NC. No commercial re-use. See rights and permissions. Published by BMJ.

${ }^{1}$ Department of Public Health and Primary Care, Katholieke Universiteit Leuven Groep Biomedische Wetenschappen, Leuven, Belgium

${ }^{2}$ Department of Cardiovascular Diseases, Universitair

Ziekenhuis Leuven Context,

Leuven, Belgium

${ }^{3}$ Institute of Health and Society, Université Catholique de Louvain (UCL), Brussels, Belgium

Correspondence to

Dr Miek Smeets;

miek.smeets@kuleuven.be

\section{ABSTRACT}

Objectives Diagnosing chronic heart failure (CHF) in general practice is challenging. Our aim was to investigate how general practitioners (GPs) diagnose CHF in real-world patients.

Design Think-aloud study.

Methods Fourteen GPs were asked to reason about four real-world CHF cases from their own practices. The cases were selected through a clinical audit. This was followed by an interview to get a deeper insight in their reasoning. The Qualitative Analysis Guide of Leuven was used as a guide in data analysis.

Results We developed a conceptual diagnostic model based on three important reasoning steps. First, GPS assessed the likelihood of CHF based on the presence or absence of HF signs and symptoms. However, this approach had serious limitations since GPs experienced many barriers in their clinical assessment, especially in comorbid elderly. Second, if CHF was considered based on step 1, the main influencing factor to take further diagnostic steps was the GPs' perception of the added value of a validated CHF diagnosis in that specific case. Third, the choice and implications of these further diagnostic steps ( $\mathrm{N}$-terminal pro B-type natriuretic peptide, ECG and/or cardiac ultrasound) were influenced by the GPs' knowledge about these tests and the quality of the cardiologists' reports.

Conclusion This think-aloud study identified the factors that influenced the diagnostic reasoning about CHF in general practice. As a consequence, targets to improve this diagnostic reasoning were withheld: a paradigm shift towards an earlier and more comprehensive risk assessment with, among others, access to natriuretic peptide testing and convincing GPs of the added value of a validated HF diagnosis.

\section{INTRODUCTION}

Heart failure (HF) is a prevalent disease that affects older patients in particular. ${ }^{2}$ The first clinical presentation usually takes place in the general practice setting. ${ }^{1}$ A distinction is made between acute and chronic HF (CHF). Early diagnosis of HF is important to initiate treatment in a timely manner and to delay progression to overt HF. ${ }^{1}$ However, a diagnosis of CHF in general practice is

\section{Strengths and limitations of this study}

This is the first study about the diagnostic reasoning of general practitioners (GPS) concerning their own real-world patients with heart failure.

- The think-aloud design is ideal to capture a sequence of thoughts involved in decision-making.

- The participating GPS were diverse in background, consistent with the general GP population; however, we did not include GPs operating in solo practice.

- The Qualitative Analysis Guide of Leuven was used as a guide to enhance the data analysis since it promotes thorough (re)reading, thinking and discussion about the research data before starting the actual coding process.

- We designed a conceptual diagnostic model and identified targets to improve the diagnostic reasoning of GPs.

challenging, leading to both underdiagnosis and overdiagnosis. ${ }^{13-6}$

Barriers affecting the diagnostic process for general practitioners (GP) were mapped by qualitative studies and showed that GPs were unfamiliar with the natural history of HF, lacked the tools (eg, cardiac ultrasound (US) and N-terminal pro B-type natriuretic peptide (NT-proBNP)) to diagnose and manage HF and were not fully aware of relevant research evidence and guidelines, despite their availability. ${ }^{78}$ Also, the GPs' need for education was expressed, as well as the importance of a holistic and chronic care approach to $\mathrm{HF}^{7}$ Additionally, GPs' reasoning when considering a diagnosis of HF was previously investigated with case-vignette studies. The objective of the latter was to compare GPs' reasoning with evidence-based guidelines. ${ }^{9}$ However, little is known about GPs' reasoning on real patients in daily practice. Gaining insight into how GPs reach a diagnosis of HF in daily practice is important, as this can provide points of action to improve the diagnostic process.

Therefore, our aim was to investigate how GPs diagnose CHF in real-world patients and 
which clinical reasoning processes are involved. Furthermore, a diagnostic model was built to capture all these concepts together.

\section{METHOD}

\section{Think-aloud method}

A method that is often used to describe the sequence of thoughts involved in decision-making is the think-aloud technique. ${ }^{10}$ Subjects are instructed to say their thoughts aloud while performing a task, and the verbal reports are usually audiotaped, transcribed to written form and then analysed. The main objective in using the think-aloud technique is not to judge the outcomes of a participant's cognitive process as either successful or unsuccessful decisions but, rather, to explore the process of performance. ${ }^{1011}$ As our goal was to unravel which arguments, barriers and facilitators play a role in the diagnosis of real-world GP patients with CHF, the think-aloud method seemed well suited to achieve this. For the methodological orientation to underpin the study, we used the constructivist grounded theory methodology as described by Glaser and Strauss. ${ }^{12} 13$ Constructivist grounded theory is an approach in which researchers generate a theory of a process, action or interaction. This theory development is shaped by or 'grounded' in the viewpoints of various participants as well as in the viewpoints of the researchers, as the latter interpret the data. ${ }^{13}$ The Consolidated Criteria for Reporting Qualitative Research was used as guidance to report our study. ${ }^{14}$

\section{Ethical considerations}

The research ethics committee of the KU Leuven approved the study (mp19078). Before the think-aloud sessions, all participant GPs were asked informed consent based on written information about the aim and methods of the study. There was no remuneration provided for participation in the study.

\section{Patient and public involvement}

No patients and/or public were involved in the development of the research question, study design or interpretation of the data.

\section{Participants}

The setting of this think-aloud study was general practice in Belgium. All general pratices in Belgium form of the public healthcare system. According to the theoretical sampling method, participating GPs and practices were selected as the analysis progressed for their ability to provide data that would confirm, challenge or expand the emerging theory. ${ }^{15}$ We aimed to include a representative sample of gender, years of practice experience, practice type and location that were consistent with the reality standards. Initially, three family practices were selected: two urban and one more rural. One of the urban practices was a district health centre that was financed at practice level and receives a fee for each registered patient.
The other practices work in a pay-for-performance system where GPs get paid for each patient who consults them. The latter is the most common system in Belgium. After 11 think-aloud sessions in these three practices, we decided to select one more rural practice with GPs not involved in academic teaching or research to guarantee a wide range of GP profiles (table 1). GPs were all approached personally or by email. All approached GPs consented to participate.

\section{Data collection}

Since patients with HF are often not registered as such in the GPs' electronic health record (EHR), we first performed a clinical audit in each EHR to identify patients with possible HF. This clinical audit consisted of the search on a registered (coded or free-text) diagnosis of HF, combined with the search on coded or free-text diagnoses of risk factors for HF, HF symptoms, and signs and combinations of HF medication (online supplementary file 1). The list of all patients with possible HF was then presented to each treating physician and they were asked to judge which patients had HF or not $(0 / 1)$ and grade how certain they were about the diagnosis (Likert scale with a range of $0 \%-10 \%-25 \%-50 \%-75 \%-90 \%-$ $98 \%$ ), based on their knowledge of the patient file. Afterwards, four patients of each GP were chosen at random for the think-aloud session: two of each binary code (HF $0 / 1$ ) and, in each category, one with a high grade of certainty (ie, $>75 \%$ ) and one with a low grade of certainty (ie, $<75 \%$ ). The order of the cases was chosen at random for all participants. The GPs were asked to think aloud about why they did or did not appoint the HF diagnosis in their own real-world patients based on the patient file in the EHR. The think-aloud session took place in the GPs' own offices. All sessions were audio recorded. The only intervention of the researcher during the thinkaloud session was that a participant who was silent for more than approximately $15 \mathrm{~s}$ was reminded to say his or her thoughts aloud about the information presented. ${ }^{10}$ The interviewer made field notes during the think-aloud session. After each think-aloud session, the participant was asked to clarify some thoughts in a follow-up interview. This whole process and the verbatim transcriptions made afterwards were led by one of the authors (PDW), a GP trainee at the time of the study. The interviewer was familiar with two of the (urban) participating practices since he was trained there. He was unfamiliar with the other two practices. As a GP trainee he had a medical background and experience as a GP, hence understanding the medical terminology and barriers associated with HF in general practice. If requested, the GPs first got a test case (not recorded), also selected at random from the audit, in order to get acquainted with the think-aloud method. Then, they continued with their four personal study cases. Data collection techniques were piloted under the supervision of a qualitative research expert (SP). The data of the test performances were not included in the study. Data collection was continued until data saturation 
Table 1 Characteristics of the participating GPs

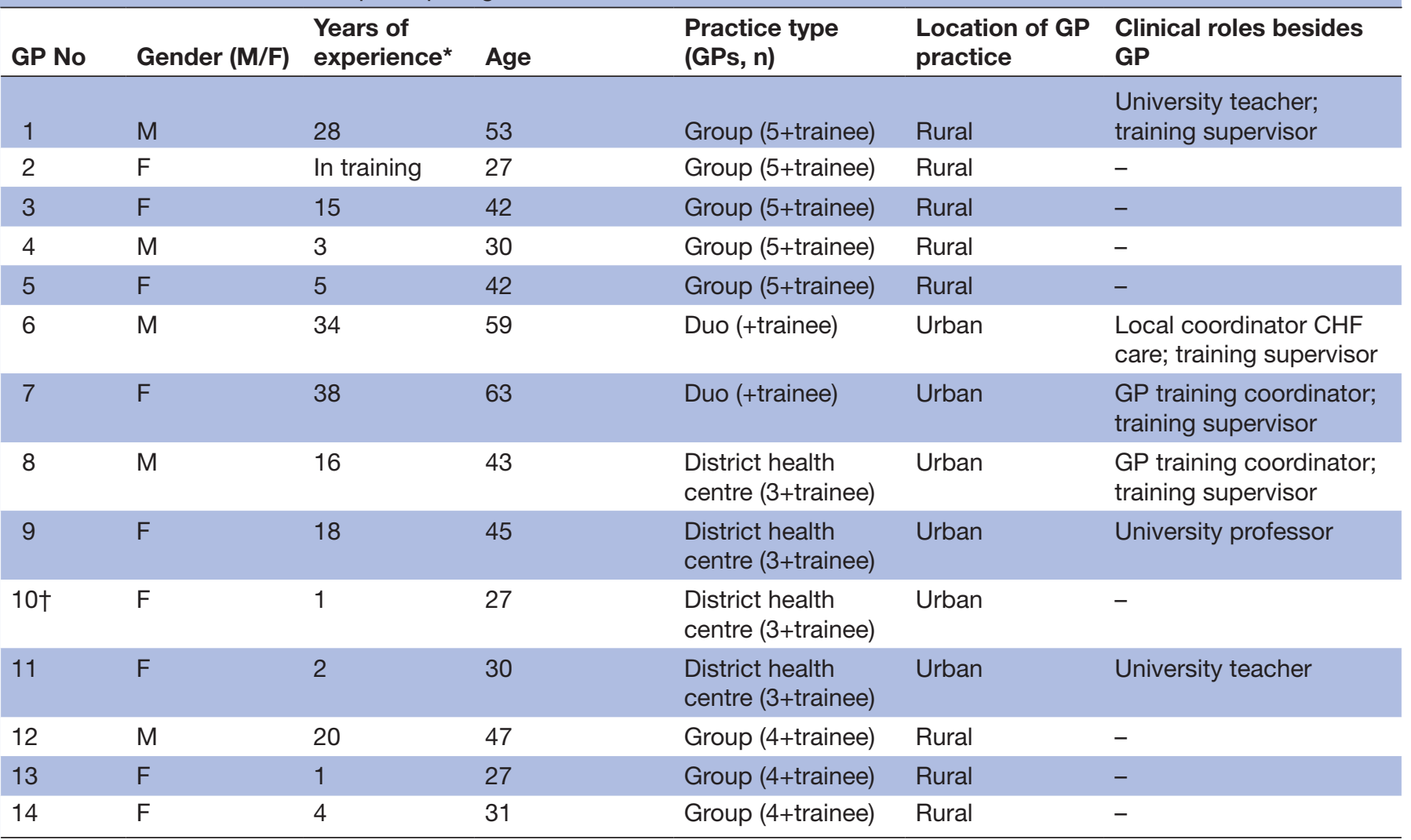

*Years in training are excluded.

tls the former GP trainee of the practice.

$\mathrm{CHF}$, chronic heart failure; GP, general practitioner

was reached. Data saturation was defined as the moment when the last two interviews no longer contributed any new elements and when a certain category had been exhaustively described in all its dimensions and variations. This means that conducting additional interviews would no longer provide new insights. ${ }^{11}$

\section{Data analysis}

The Qualitative Analysis Guide of Leuven (QUAGOL) was held as a guide for the data analysis. ${ }^{16}$ The QUAGOL is a theory and practice-based guide that offers a comprehensive method to guide the process of qualitative data analysis within the grounded theory approach. It promotes thorough (re) reading, thinking and discussion about the research data before starting the actual coding process. ${ }^{16}$ All transcripts were entered into NVivo V.11 software (QSR International, Melbourne, Australia), a data management platform, for qualitative data analysis. After two of the authors (PDW and MS-a GP specialised in HF) familiarised themselves with the data by making one-sheet summaries to aid categorisation and conceptualisation, they independently coded each line of text according to its meaning and content. Codes were created inductively. After reading and coding the findings of a sample of transcripts, the two researchers discussed and compared the codes for similarities and differences until a primary coding framework was constructed. Subsequently, the findings of the other transcripts were independently read and coded. The two authors discussed their respective coding frameworks frequently to reach consensus. Codes were added, modified or merged when necessary. This process resulted in a tree structure with several layers for organising the descriptive themes (online supplementary file 2). From these, a set of analytical themes emerged that were discussed by the research team. Our collaborative approach and the iterative constant comparison limited the extent to which individual perspectives or background could dominate our interpretation. ${ }^{13}$

\section{RESULTS}

The 14 participants had been specialists in family medicine for a median of 10 years (IQR 1.8-22). Their mean age was $40 \pm 13$ years old, and 9 of them were women $(64 \%)$ (table 1). The length of the think-aloud session and follow-up interview was, on average, $27 \mathrm{~min}$ (range 22-42). We summarised our findings in a conceptual diagnostic model (figure 1).

\section{Step 1: assessing the likelihood of HF}

Implicitly, every GP assessed how likely HF was in their patient. To estimate the likelihood, many factors were 


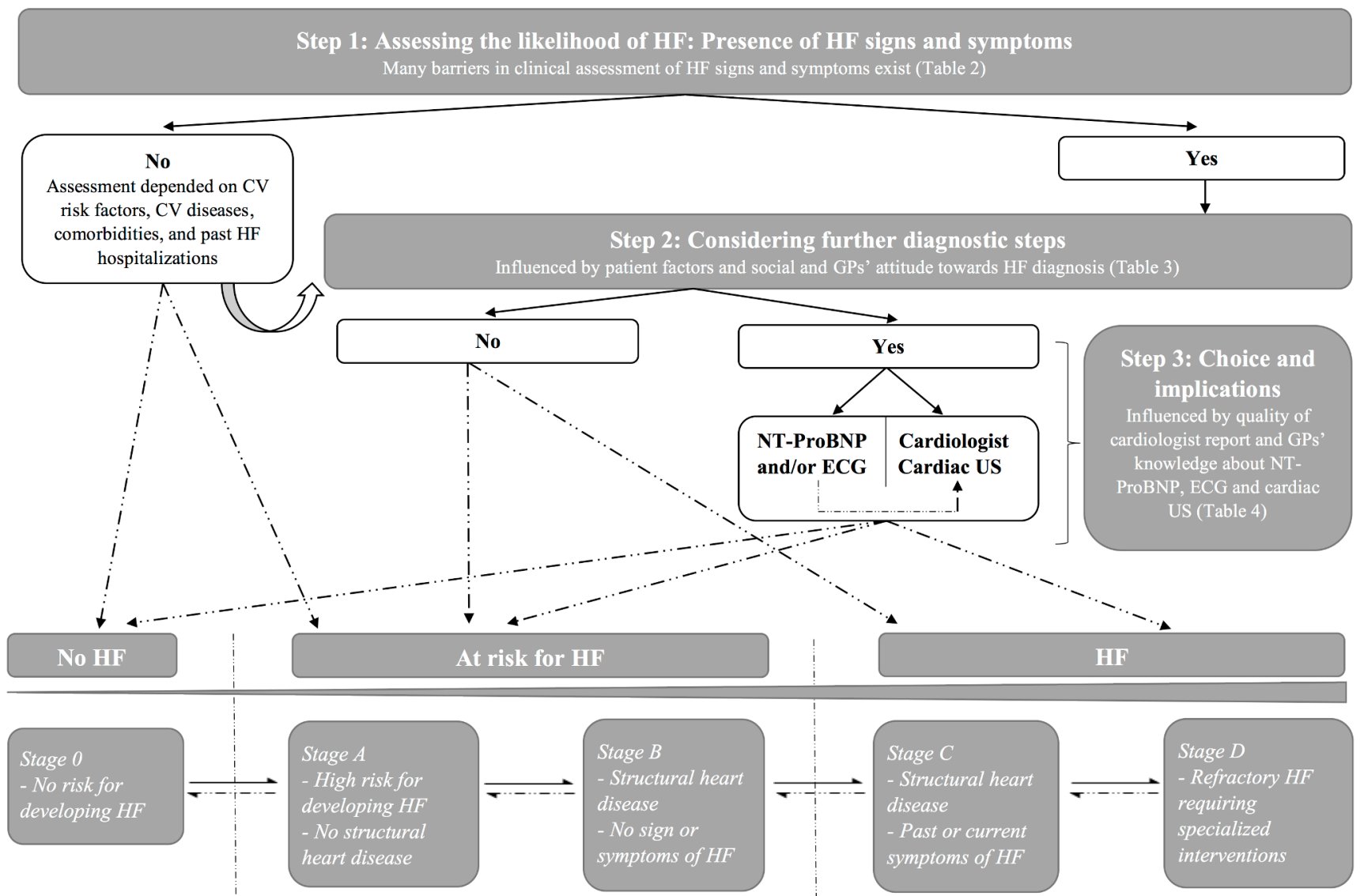

Figure 1 Diagnostic reasoning model. CV, cardiovascular; GP, general practitioner; HF, heart failure; NT-proBNP, N-terminal pro B-type natriuretic peptide; US, ultrasound.

considered, such as cardiovascular (CV) risk factors, $\mathrm{CV}$ diseases and medication, comorbidities, past HF hospitalisations, and HF signs and symptoms. In patients without any or few risk factors, GPs considered HF unlikely (figure 1).

He is 43 and is still a young man. As far as I know, something cardiac never occurred to him. I can also see this in his antecedents list. He never had any complaint that I can link with HF like dyspnoea, oedema, etc. So, actually, I am not $98 \%$, but $100 \%$ sure he doesn't have HF! (GP 3, Think-aloud session)

In patients with a CV history, the presence of HF signs and symptoms was cited as the most important discriminator to distinguish between being at risk for HF and having HF (figure 1). Consequently, for almost all GPs, the clinical assessment determined the further diagnostics process and their risk assessment.

If a patient has symptoms we look further but without symptoms we don't. (...) In my opinion, the clinical aspect is the most important. For instance, the oede$\mathrm{ma}$, the gain of weight and the minimized exercise capacity. (GP 14, Follow-up interview)

However, many GPs recognised barriers in this clinical approach (table 2). It was apparent that almost every GP

experienced difficulties caused by overlap in signs and symptoms with comorbidities in these real-world cases.

Yes, of course, there are many overlapping symptoms. She suffers from chronic hypoxia, and this is causing her fatigue. Her limited exercise tolerability, dyspnoea, cough and abnormal lung auscultation can also be caused by this. The core symptoms are overlapping, which makes it very difficult to appoint a clear (HF) diagnosis to someone with chronic obstructive lung disease on clinical grounds. (GP 4, Follow-up interview)

Even without concurrent comorbidities, it remained difficult to assess patients clinically due to the non-specificity of HF signs and symptoms.

Table 2 Assessing the likelihood of HF-influencing factors

Barriers in the assessment of HF symptoms and signs

- Non-specificity of some symptoms and signs

- Masked by medication

- Difficult in immobile patients

- Relapsing remitting course

$H F$, heart failure. 
Table 3 Considering further diagnostic steps-influencing factors

$\begin{array}{ll}\text { Patient and social } & \text { Attitude towards diagnosis, follow-up } \\ \text { factors } & \text { and treatment } \\ & \text { Lifestyle, self-care and compliance } \\ & \text { Choice for a palliative care approach } \\ & \text { Age, frailty and impact of stay in a } \\ & \text { long-term care facility } \\ & \text { Length of GP-patient relationship } \\ & \text { Language barrier } \\ & \text { Comorbidities that influence clinical } \\ & \text { assessment } \\ & \text { Perceived value of cardiologist } \\ & \text { referral and an objectified HF } \\ & \text { diagnosis regarding: } \\ & - \text { Implications for further treatment } \\ & - \text { GPs' priorities } \\ \text { GP factors } & \text { Dealing with diagnostic uncertainty } \\ & \text { Diastolic versus systolic HF }\end{array}$

GP, general practitioner; HF, heart failure.

She had a little peripheral oedema in summer. Is this linked with HF? I really don't know. (GP 2, Follow-up interview)

Additionally, many patients already received CV medication, possibly masking HF symptoms and signs.

It could always be possible that he has a slight aspect of HF and because of the ACE-inhibitor he takes it is just masked. That it suffices to control his symptoms.

(GP 7, Think-aloud session)

Other barriers mentioned were difficulties to assess the HF risk in immobile patients, which was often the case in this population of elderly; the relapsing remitting course of $\mathrm{HF}$ was also a challenge (table 2).

\section{Step 2: considering further diagnostic steps}

The decision about further diagnostic steps was influenced by patient and social factors and by the GPs' attitudes towards HF diagnosis (table 3).

\section{Patient and social factors}

GPs tended to choose a merely clinical approach without technical investigations in palliative care situations and in patients living in long-term care facilities. Old age and frailty were also patient factors GPs considered to choose such an approach.

Well, mostly, there is a good reason why someone arrives at a long-term care facility. Because living alone at home is arduous, excursions are getting difficult or dementia is progressing. The need for care increases, and the drive to do technical investigations decreases. The benefits for the patient are limited compared to the efforts that these investigations require. And, besides, if you visit a cardiologist, it is rarely one investigation, and I think it would be too big of a burden for him. (GP 12, Follow-up interview)

Thereby, the patients' own attitude towards diagnosis, follow-up and treatment was seen as an influencing factor for GPs to reject or favour further diagnostic steps. Additionally, patients' lifestyle, self-care and compliance were seen as fundamental elements to sustain this attitude.

I think this patient will ask for further investigations herself because she is a worried person and wants rather too many than too few technical investigations. (GP 11, Follow-up interview)

I have the impression that this lady is fed up with all medical follow-up. She sighs very deeply when I want to refer her. Therefore, it is important for me to have an eye on her. (GP 2, Follow-up interview)

A language barrier, a short length of the GP-patient relationship and masking comorbidities lowered the threshold for further diagnostic steps. GPs had less trust in their clinical assessment in these cases.

Because of the language barrier, it is very hard to do a proper history. Consequently, he only comes with his complaints, and he is unable to answer my questions. Combined with his cardiovascular risk profile, I consider him even more at risk because I feel I don't have any control on his situation. (GP 8, Think-aloud session)

\section{GPs' attitudes towards further diagnostic steps}

Clearly, GPs' attitudes towards further investigations also influenced clinical decision-making. An important consideration was the potential prognostic benefit of an objectified HF diagnosis.

In this case, a cardiologist's referral could be definitely interesting. She is only 70 years old, and making the correct diagnosis could be very important for her prognostically. (GP 1, Follow-up interview)

Conversely this was also mentioned as a barrier for referral.

It has been a while since this patient visited a cardiologist. I might consider referring him again, but, on the other hand, I am wondering: 'if we can label him with an HF diagnosis, would this change anything for his current medical treatment and life expectancy?' (GP 7, Think-aloud session)

GPs tended to set out personal priorities for each patient with multimorbidity, emphasising the patient's needs for well-being.

The question is if it is a priority to diagnose HF. For this man, 76 years old but biologically older, I am wondering if you shouldn't aim for what is really important to him. He has a lot of pain and doesn't see the connection with lack of exercise because of his dementia. (...) What is the priority for this patient? 
In my opinion, it is not the diagnosis but being more active. (GP 6, Follow-up interview)

In addition, differences were noted in the way GPs dealt with diagnostic uncertainty. Some GPs always strived for objectified diagnoses and only accepted uncertainty in exceptional circumstances, while other GPs obviously felt more comfortable with a certain degree of uncertainty and were more reluctant to refer. This attitude was not linked to age or practice type but was rather linked to the GP's personality.

The concept of categorisation based on ejection fraction (EF) in $\mathrm{HF}$ and the therapeutic implications of an $\mathrm{HF}$ with reduced $\mathrm{EF}$ (HFrEF) diagnosis versus an $\mathrm{HF}$ with preserved EF (HFpEF) diagnosis were not always known by the participating GPs. Although, for those who were aware of the difference, it did influence their decision-making.

This is an example where the clinical diagnosis was very obvious and, at this moment, you start making considerations. She is a single, 87 years old, less mobile elderly women; what is the added value of objectifying your diagnosis? And, in particular, does this make any prognostic difference for her? In my opinion, this is the prototype of diastolic HF: the obese, elderly women where, from a prognostic view, not so much could be gained. An ACE-inhibitor and a beta-blocker-by the way, she already takes one-don't have any prognostic importance. It is just controlling the symptoms with diuretics. So, at this point of view, I am not going to bother her with cardiologist referrals for my own wish for certainty. (GP 1, Follow-up interview)

\section{Step 3: choice and implications of further diagnostic steps}

When GPs chose to refer, they almost immediately opted for a cardiologist and/or cardiac US referral (figure 1). GPs rarely mentioned ECG or NT-proBNP spontaneously. Influencing factors on how they decided and dealt with these investigations and their results were described in table 4 .

\section{NT-proBNP and/or ECG as diagnostic tests in HF $\mathrm{N}$-terminal pro B-type natriuretic peptide}

NT-proBNP as a diagnostic test was rarely mentioned during the think-aloud session. The views of the GPs were further explored in the interviews afterwards. First, almost every GP considered the fact that the test is not reimbursed as an important barrier.

I like using the test, or, better, I would like using it. The problem is that in ambulatory practice the test is not reimbursed. For me this is a big obstacle to use it systematically in my daily routine. (GP 12, Follow-up interview)

Most GPs knew NT-proBNP is a good marker to exclude HF but possible other indications were not very clear.
Table 4 Choice and implications of further diagnostic steps-influencing factors

\begin{tabular}{|c|c|}
\hline NT-proBNP & $\begin{array}{l}\text { Price as a barrier, demand for } \\
\text { reimbursement } \\
\text { - Utility (not) known } \\
\text { - Interpretation problems }\end{array}$ \\
\hline $\begin{array}{l}\text { NT-proBNP and } \\
\text { ECG }\end{array}$ & $\begin{array}{l}\text { Perception of positive and negative } \\
\text { predictive value } \\
\text { Integrated in workflow } \\
\text { Uncertainty about indication }\end{array}$ \\
\hline $\begin{array}{l}\text { Cardiologist and } \\
\text { cardiac US }\end{array}$ & $\begin{array}{l}\text { Perception of positive and negative } \\
\text { predictive value } \\
\text { GPs' knowledge about cardiac US } \\
\text { - Quality of cardiologist report } \\
\text { - Confirmation of HF diagnosis by } \\
\text { cardiologist } \\
\text { - Remaining diagnostic uncertainty } \\
\text { after cardiologist appointment } \\
\text { Clinical assessment of HF by } \\
\text { cardiologist and mutual trust } \\
\text { Importance of cardiorenal } \\
\text { consultation } \\
\text { - HFpEF as a new difficult entity }\end{array}$ \\
\hline
\end{tabular}

GP, general practitioner; HF, heart failure; HFpEF, heart failure with preserved ejection fraction; NT-proBNP, N-terminal pro B-type natriuretic peptide; US, ultrasound.

The cardiologist once asked me to measure it (NTProBNP) again. The rationale behind this, to control the value in follow-up, is not clear to me. It is an excellent parameter to exclude rather than prove HF. I can imagine when someone is less decompensated, there will be less stretch on the heart, and the parameter will fall, but I don't know if it is a good parameter to follow the severity of heart failure. I rather think it isn't. (GP 4, Follow-up interview)

Although there were GPs who did not see the benefit of the test at all, almost all GPs admitted the test simply was not integrated in their workflow.

(NT-pro)BNP? No, however, I was involved in academic research of the subject; I must admit it is not accustomed in my flow yet. (GP 9, Follow-up interview)

Some GPs acknowledged they were not always sure how to interpret the results.

I am not so sure about its cut-off values. In my opinion, it is something vague. You might say it is elevated or not, but it remains difficult to interpret. (GP 14, Follow-up interview)

\section{Electrocardiography}

If GPs mentioned an ECG, it was mostly done by the cardiologist rather than being one they performed themselves. Whether it could be helpful in the assessment of HF differed between all participating GPs. 
An ECG, if I am right, is a test which is very sensible for HF, but not specific. So, ideal to exclude but hard to prove. According to me, a perfectly normal ECG excludes HF. But, in this case, she has an AF, so I already know what to expect. (GP 4, Follow-up interview)

\section{Cardiologist and cardiac US}

Different attitudes towards the positive and negative predicted value of cardiac US were noted. The negative predictive value of a normal cardiac US was widely accepted. However, the perception of the need and positive predictive value differed between the participating GPs.

I always make decisions based on clinical grounds and never according to an aberrant cardiac ultrasound. (GP 14, Follow-up interview)

But, here I notice I really need the cardiac ultrasound to exclude HF, while for demonstrating HF I don't need that anymore. (GP 8, Follow-up interview)

I just like having a cardiac ultrasound because it is very clear to me. (GP 5, Think-aloud session)

Attitudes towards the value of a cardiologist referral and the interpretation of the cardiac US results depended on the quality of the cardiologist's report and the GPs' knowledge about cardiac US. GPs reported frustrations about the lack of an EF or a confirmed HF diagnosis in the reports from the cardiologists and suggested that explicitly asking for it could help.

The cardiologists' reports are often not that great. It happens that they don't mention the EF and are not giving any information about it at all. I must admit it is better now than last year's, and it is a bit cardiologist-dependent. But, it could be vexing because you would like to advise your patients what they should do. (GP 12, Follow-up interview)

You should ask it (HF) particularly because sometimes they (cardiologists) remain silent about it. Often HF isn't mentioned in the cardiologists' conclusion, while it is a very important risk factor for hospitalization and mortality. (GP 4, Follow-up interview)

Some GPs reported a lack of mutual trust in the collaboration with cardiologists.

Hardly one listens to the patient or the GP. A flare that doesn't take place in the hospital is considered to be a non-existing flare. (GP 8, Follow-up interview)

Those GPs who were aware of the difference between HFrEF and HFpEF acknowledged that an HFrEF diagnosis was easier to assign than an HFpEF diagnosis. If a cardiologist did not confirm the HFpEF diagnosis in symptomatic patients, diagnostic doubt remained for most GPs. Some GPs who are very experienced in HF drew their own conclusions based on the clinical image and cardiac US report.
What you see by these type of patients is that cardiologists aren't either always able to recognize this clinical image as HF with preserved EF, while, in this case, you have several clinical and even some echocardiographic arguments. (GP 6, Follow-up interview)

Remaining diagnostic doubts after referral had a negative influence on the GPs' attitudes towards referral. A cardiorenal consultation was seen as a big advantage because of the multifactorial approach needed for HF (table 4).

\section{DISCUSSION}

This think-aloud study highlights the influencing factors in the diagnostic reasoning process of CHF in general practice. A conceptual diagnostic model was built to capture the three main diagnostic reasoning steps. First, GPs assessed the likelihood of HF using the presence or absence of HF signs and symptoms as the main discriminating factor. However, this approach had serious limitations since GPs experienced many barriers in their clinical assessment, especially in comorbid elderly. Second, if CHF was considered based on step 1, the main influencing factor to take further diagnostic steps was the GPs' perception of the added value of a validated CHF diagnosis in that specific case. Third, the choice and implications of these further diagnostic steps (NT-proBNP, ECG and/or cardiac US) were influenced by the GPs' knowledge about these tests and the quality of the cardiologists' reports.

\section{Step 1: assessing the likelihood of HF}

Every GP assessed the likelihood of HF as a first step. The arguments GPs used in their assessment coincided with the concept of the CV continuum or the HF stages of the American Heart Association guideline. These concepts describe $\mathrm{HF}$ as a syndrome that progresses from asymptomatic structural heart disease in patients with $\mathrm{CV}$ risk factors to symptomatic HF, making this likelihood assessment a valuable approach. ${ }^{17}{ }^{18}$ Additionally, in line with former studies, the assessment of HF signs and symptoms by the GP was seen as the main discriminating factor to withhold a diagnosis of $\mathrm{HF}$ and/or to consider further diagnostic steps. ${ }^{79}$ However, at the same time, GPs reported many barriers in this clinical approach, especially in real-world older patients with comorbidities, as confirmed in other studies. ${ }^{56}$ Furthermore, it was shown that $77 \%$ of the patients with HF diagnosed in primary care in Belgium are already in New York Heart Association (NYHA) stages III-IV and thus unmistakably symptomatic at the time of diagnosis. ${ }^{19}$ Consequently, one might suspect that GPs tend to overestimate the value of their clinical assessment, especially in older patients with HF, leading to delayed or missed diagnoses. ${ }^{4-6} 20$ This provides important points of action to improve the diagnostic process in HF. First, a paradigm shift is needed towards early 
identification of patients with $\mathrm{HF}$ and prevention of disease progression. Early intervention in patients with stage B HF (asymptomatic structural heart disease) led to a reduction in morbidity and mortality. ${ }^{21}$ Therefore, we would like to emphasise the importance to consider further diagnostic steps when signs and symptoms are present and when substantial risk factors or comorbidities are known (figure 1, curled arrow) ${ }^{22}{ }^{23}$ Second, the question arises whether the current definition of HF is applicable in the very old. The definition of HF restricts itself to stages at which clinical symptoms are apparent. As shown by our study, this is particularly difficult in elderly as signs and symptoms lose their value in this age group. Demonstrating an underlying cardiac cause is another essential part of the HF definition. This is especially challenging in patients with HFpEF. A high proportion of patients with HFpEF have concurrent atrial fibrillation while diastolic dysfunction is very difficult to assess in this patient group. ${ }^{24}{ }^{25}$ Additionally, some patients only exhibit symptoms (and echocardiographic abnormalities) on exertion. ${ }^{24}{ }^{25}$ Conversely, the prevalence of mild to moderate diastolic dysfunction is very high in elderly but it is difficult to decide on the clinical significance of these cardiac phenotypes. ${ }^{6} 2526$ In response to these diagnostic problems, the Heart Failure Association introduced a new consensus on the HFpEF diagnosis on the latest European Society of Cardiology (ESC) congress. ${ }^{27}$ Time will tell whether this new HFpEF definition will resolve all diagnostic doubts.

\section{Step 2: considering further diagnostic steps}

Our study revealed patient-related, social and GP-related factors that were not reproducible by case-vignette studies $^{9}$ or discussed in HF guidelines. ${ }^{1} 18$ Patient-related and social factors are generally not modifiable; however, GP-related factors are. The importance GPs attach to person-centred care was highlighted. As many GPs mentioned in our study, what matters to them and to the patients is the prognostic and therapeutic implications of cardiac abnormalities. This is an issue that HF guideline developers should consider. ${ }^{18}$ NT-proBNP could support GPs in this risk stratification, as it provides prognostic information in cardiac outpatients. ${ }^{1} 3202829$ Additionally, in our study, the concepts of HFrEF and HFpEF were cited by very few GPs. Interestingly, some GPs thought that they could distinguish HFrEF from HFpEF based on the patients' clinical profile, while cardiac US remains the gold standard. ${ }^{18}$ Therefore, we recommend that GPs have a better understanding of cardiac US reports and propose targeted education as an area of improvement. ${ }^{730}$

\section{Step 3: choice and implications of further diagnostic steps}

NT-proBNP and ECG are recommended by all HF guidelines. ${ }^{18}$ Conversely, they were rarely mentioned by GPs in this think-aloud study. In the follow-up interview, the lack of reimbursement for NT-proBNP tests was cited as a significant barrier. In Belgium, the cost of natriuretic peptide testing is relayed on the patient $( \pm € 25$ per test) due to an impasse in the negotiations with clinical biologists. This accounted for the GPs' unfamiliarity with the test and the uncertainty regarding how to integrate it in their practice. However, one GP correctly quoted the value of NT-proBNP in the early stages of HF but voiced scepticism towards extended use in follow-up. ${ }^{31}$ Furthermore, the cost of the test could account, in part, for the fact that $77 \%$ of Belgian patients with $\mathrm{HF}$ in primary care are already in NYHA stages III-IV at the time of HF diagnosis. ${ }^{19}$ To withhold ECG as a valuable diagnostic tool, repeated training of GPs to finetune their interpretation skills remains important. ${ }^{32}$ However, as quoted pertinently in our study, a negative ECG excludes HF, but the elderly rarely have a completely normal ECG. ${ }^{16}$ Our study also showed that GPs sought more information on the correct interpretation of cardiac US reports when diagnosing CHF. In contrast with UK studies, there were few practical barriers for cardiologist referrals and cardiac US. ${ }^{783}$ Thus, better access alone to cardiac US is not sufficient because a better understanding of and education in interpreting cardiologists' reports is needed. ${ }^{89}$ Additionally, GPs noted that cardiologists are responsible to describe HF diagnoses clearly in their reports, which, in the case of HFpEF, remains a difficult task. ${ }^{124}$

\section{Implications for practice}

The diagnostic flow chart of the ESC HF guideline already promotes using NT-proBNP or BNP as a diagnostic test when patients have a history of ischaemic heart disease, hypertension, cardiotoxic medication or chronic diuretic use. ${ }^{1}$ Belgian GPs rather use the national GP guideline about CHF published in 2011. Recommendations are generally in line with the ESC HF guideline but the use of natriuretic peptides is not actively promoted in this guideline since the test is not reimbursed in Belgium. ${ }^{34}$ The paradigm shift to an earlier risk assessment should be more widely disseminated in practice. However, access to natriuretic peptides is indispensable to achieve this. ${ }^{1222829}$ The main modifiable barrier for further investigations in this study is not, as formerly described, a lack of access or a pure lack of knowledge. It is a lack of belief in the added value of further investigations. A remaining uncertainty after cardiologist referral contributes to this. Uncertainty remains because cardiologists do not assign HFpEF diagnoses easily themselves, and GPs are not able to correctly interpret echocardiography reports. ${ }^{35}$ Additionally, GPs are confronted with a high percentage of patients with HFpEF, whose prognosis does not change much with available treatment. Therefore, assigning a diagnosis in this patient group seemed less important in their opinion. Education and guidelines for GPs should target these beliefs because a distinction between HFrEF and HFpEF cannot always be made clinically and a correct HFpEF diagnosis does have important prognostic implications. ${ }^{36}$ 


\section{Methodological strengths and limitations of this study}

The variability in the years of experience, the nature of the practice and the gender of the 14 participating GPs reflected the general GP population. Therefore, differences in working conditions, access to cardiac US and cooperation with specialists were well covered. However, there were no solo-operating GPs included in the study. This form of practice is declining but is currently still represented in the Belgian healthcare system. In this survey, there was also a higher fraction of participant GPs involved in academic teaching and/or research, explaining our recruitment success rate of $100 \%$. To correct for this imbalance, one extra rural practice with three GPs who were not involved in academic teaching or research was added in the study. As far as we know, this is the first study where a think-aloud approach on real-world patients was performed. This enabled us to highlight the importance GPs attach to person-centred care and to analyse GPs' diagnostic reasoning with respect to CHF and the differences with the existing guidelines.

\section{CONCLUSION}

This think-aloud study identified the influencing factors in the diagnostic reasoning process of $\mathrm{HF}$ in general practice. As a consequence, targets to improve this diagnostic reasoning were identified: a paradigm shift towards earlier risk assessment, rethinking the HF definition in the very old, promoting access to NT-proBNP and convincing GPs of the added value of an objectified HF diagnosis through a better cooperation with cardiologists.

Acknowledgements The authors thank all the participating general practitioners.

Contributors MS, PDW, SP and BV are responsible for the design of the study. PDW performed the audit and think-aloud interviews in each practice. MS and PDW analysed the data and wrote the manuscript. All authors (MS, PDW, SP, BV, BA and SJ) revised the manuscript.

Funding The authors have not declared a specific grant for this research from any funding agency in the public, commercial or not-for-profit sectors.

Competing interests None declared.

Patient consent for publication Not required.

Ethics approval The research ethics committee of the KU Leuven approved the study (mp19078) on 30 March 2017.

Provenance and peer review Not commissioned; externally peer reviewed.

Data sharing statement All authors had full access to all data in the study and assume responsibility for the integrity of the data and the accuracy of the data analyses.

Open access This is an open access article distributed in accordance with the Creative Commons Attribution Non Commercial (CC BY-NC 4.0) license, which permits others to distribute, remix, adapt, build upon this work non-commercially, and license their derivative works on different terms, provided the original work is properly cited, appropriate credit is given, any changes made indicated, and the use is non-commercial. See: http://creativecommons.org/licenses/by-nc/4.0/.

\section{REFERENCES}

1. Ponikowski P, Voors AA, Anker SD, et al. 2016 ESC Guidelines for the diagnosis and treatment of acute and chronic heart failure: The Task
Force for the diagnosis and treatment of acute and chronic heart failure of the European Society of Cardiology (ESC). Developed with the special contribution of the Heart Failure Association (HFA) of the ESC. Eur J Heart Fail 20162016;18:891-975.

2. Bleumink GS, Knetsch AM, Sturkenboom MC, et al. Quantifying the heart failure epidemic: prevalence, incidence rate, lifetime risk and prognosis of heart failure The Rotterdam Study. Eur Heart J 2004;25:1614-9.

3. Bhatia GS, Sosin MD, Stubley J, et al. Evaluation of B-type natriuretic peptide for validation of a heart failure register in primary care. $B M C$ Cardiovasc Disord 2007;7:23.

4. Valk MJ, Mosterd A, Broekhuizen BD, et al. Overdiagnosis of heart failure in primary care: a cross-sectional study. $\mathrm{Br} J$ Gen Pract 2016;66:e587-e592.

5. Hancock HC, Close H, Mason JM, et al. High prevalence of undetected heart failure in long-term care residents: findings from the Heart Failure in Care Homes (HFinCH) study. Eur J Heart Fail 2013;15:158-65.

6. Smeets M, Degryse J, Aertgeerts B, et al. General practitioners' judgement of chronic heart failure in the oldest old: Insights from the BELFRAIL study. Int J Cardiol 2015;191:120-7.

7. Smeets M, Van Roy S, Aertgeerts B, et al. Improving care for heart failure patients in primary care, GPs' perceptions: a qualitative evidence synthesis. BMJ Open 2016;6:e013459.

8. Hancock HC, Close H, Fuat A, et al. Barriers to accurate diagnosis and effective management of heart failure have not changed in the past 10 years: a qualitative study and national survey. BMJ Open 2014;4:e003866.

9. Skånér $\mathrm{Y}$, Backlund L, Montgomery H, et al. General practitioners' reasoning when considering the diagnosis heart failure: a think-aloud study. BMC Fam Pract 2005;6:4.

10. Ericsson KA SH. Protocol analysis: verbal reports as data, 1980.

11. O'Reilly M, Parker N. 'Unsatisfactory Saturation': a critical exploration of the notion of saturated sample sizes in qualitative research. Qualitative Research 2013;13:190-7.

12. Glaser BG, Strauss AL. The discovery of grounded theory: Strategies for qualitative research. Chicago: Aldine Publishing Co, 1967.

13. Charmaz K. Constructing grounded theory: A practical guide through qualitative analysis. London, UK: Sage, 2006.

14. Tong A, Sainsbury P, Craig J. Consolidated criteria for reporting qualitative research (COREQ): a 32-item checklist for interviews and focus groups. Int J Qual Health Care 2007;19:349-57.

15. Watling CJ, Lingard L. Grounded theory in medical education research: AMEE Guide No. 70. Med Teach 2012;34:850-61.

16. Dierckx de Casterlé B, Gastmans C, Bryon E, et al. QUAGOL: a guide for qualitative data analysis. Int J Nurs Stud 2012;49:360-71.

17. Dzau V, Braunwald E. Resolved and unresolved issues in the prevention and treatment of coronary artery disease: a workshop consensus statement. Am Heart J 1991;121(4 Pt 1):1244-63.

18. Yancy CW, Jessup M, Bozkurt B, et al. 2013 ACCF/AHA guideline for the management of heart failure: a report of the American College of Cardiology Foundation/American Heart Association Task Force on practice guidelines. Circulation 2013;128:e240-e327.

19. Devroey D, Van Casteren V. The incidence and first-year mortality of heart failure in Belgium: a 2-year nationwide prospective registration. Int J Clin Pract 2010;64:330-5.

20. Barents M, van der Horst IC, Voors AA, et al. Prevalence and misdiagnosis of chronic heart failure in nursing home residents: the role of B-type natriuretic peptides. Neth Heart J 2008;16:123-8.

21. Subzposh F, Gupta A, Hankins SR, et al. Management of ACCF/AHA Stage A and B patients. Cardiol Clin 2014;32:63-71.

22. Ledwidge M, Gallagher J, Conlon C, et al. Natriuretic peptide-based screening and collaborative care for heart failure: the STOP-HF randomized trial. JAMA 2013;310:66-74.

23. Di Bello V, La Carrubba S, Antonini-Canterin F, et al. Role of electrocardiography and echocardiography in prevention and predicting outcome of subjects at increased risk of heart failure. Eur J Prev Cardiol 2015;22:249-62.

24. Rutten FH, Clark AL, Hoes AW. How big a problem is heart failure with a normal ejection fraction? BMJ 2016;353:i1706.

25. Nagueh SF, Appleton CP, Gillebert TC, et al. Recommendations for the evaluation of left ventricular diastolic function by echocardiography. Eur J Echocardiogr 2009;10:165-93.

26. Ammar KA, Jacobsen SJ, Mahoney DW, et al. Prevalence and prognostic significance of heart failure stages: application of the American College of Cardiology/American Heart Association heart failure staging criteria in the community. Circulation 2007; 115:1563-70.

27. Pieske $B$. Diagnosing heart failure with preserved ejection fraction: the new HFA consensus. 2018. https://www.escardio.org/ Congresses-\&-Events/Heart-Failure/Congress-resources/News/ 
diagnosing-heart-failure-with-preserved-ejection-fraction-the-newhfa-consensus (updated 26 May 2018).

28. Smeets M, Degryse J, Janssens S, et al. Diagnostic rules and algorithms for the diagnosis of non-acute heart failure in patients 80 years of age and older: a diagnostic accuracy and validation study. BMJ Open 2016;6:e012888.

29. Toggweiler S, Borst O, Enseleit F, et al. NT-proBNP provides incremental prognostic information in cardiac outpatients with and without echocardiographic findings. Clin Cardiol 2011;34:183-8.

30. Peters-Klimm F, Campbell S, Müller-Tasch T, et al. Primary carebased multifaceted, interdisciplinary medical educational intervention for patients with systolic heart failure: lessons learned from a cluster randomised controlled trial. Trials 2009;10:68.

31. Felker GM, Anstrom KJ, Adams KF, et al. Effect of Natriuretic PeptideGuided Therapy on Hospitalization or Cardiovascular Mortality in High-Risk Patients With Heart Failure and Reduced Ejection Fraction: A Randomized Clinical Trial. JAMA 2017;318:713-20.
32. Lim TK, Collinson PO, Celik E, et al. Value of primary care electrocardiography for the prediction of left ventricular systolic dysfunction in patients with suspected heart failure. Int $J$ Cardiol 2007;115:73-4.

33. Cleland JG, Cohen-Solal A, Aguilar JC, et al. Management of heart failure in primary care (the IMPROVEMENT of Heart Failure Programme): an international survey. Lancet 2002;360:1631-9.

34. Van Royen P, Boulanger S, Chevalier P, et al. Aanbeveling voor goede medische praktijkvoering: Chronisch hartfalen. Huisarts $\mathrm{Nu}$ 2011;40:158-86.

35. Deaton C, Edwards D, Malyon A, et al. The tip of the iceberg: finding patients with heart failure with preserved ejection fraction in primary care. An observational study. BJGP Open 2018;2.

36. Dunlay SM, Roger VL, Redfield MM. Epidemiology of heart failure with preserved ejection fraction. Nat Rev Cardiol 2017;14:591-602. 